\title{
Maternal and fetal levels of methionine and homocysteine in early human pregnancy
}

\author{
${ }^{*} \dagger$ Régine P. M. Steegers-Theunissen Clinical epidemiologist, **Neville C. Wathen Consultant, \\ $\left\lceil\right.$ Tom K. A. B. Eskes Professor, \$Bertie van Raaij-Selten Laboratory technician, ${ }^{* *}$ Tim Chard Professor \\ Departments of *Epidemiology, †Obstetrics and Gynaecology and $\ddagger$ Paediatrics, University Hospital Nijmegen, The Netherlands; \\ **Fetal Medicine Unit, Homerton Hospital, London, UK
}

Objective To investigate methionine metabolism during normal human embryonic development by measuring levels of methionine and total homocysteine in samples of maternal serum, extra-embryonic coelomic fluid, and amniotic fluid.

Design Cross-sectional observational study.

Setting Collaboration between St Bartholomew's Hospital, London, and the University Hospital of Nijmegen in The Netherlands.

Participants Twenty-three women with uncomplicated pregnancies between 8 and 12 weeks of gestation before surgical termination of an ultrasonographically normal fetus.

Methods Maternal serum samples were collected prior to surgery. Samples of extra-embryonic fluid and amniotic fluids were obtained by transvaginal ultrasound-guided coelocentesis and amniocentesis. Methionine was measured using an aminoacid analyser and total homocysteine by high performance liquid chromatography.

Results Levels of methionine were four times higher in extra-embryonic coelomic fluid and twice as high in amniotic fluid compared with maternal serum. In contrast, the total homocysteine concentrations were much lower in both extra-embryonic coelomic fluid and amniotic fluid than in maternal serum. All differences were significant $(P \leq 0 \cdot 01)$.

Conclusions The comparatively high concentrations of methionine in extra-embryonic coelomic fluid and amniotic fluid, and the concomitant low levels of total homocysteine in these fluids, suggest a role for methionine metabolism during early human pregnancy.

\section{INTRODUCTION}

The mechanisms involved in the nutrition of the embryo and fetus are poorly understood. The dynamic and physiology of the transplacental transport of the amino acid methionine, its derivative homocysteine, folate and vitamin $B_{12}$ which are involved in homocysteine remethylation (Fig. 1) has been studied previously by us in the second and third trimester of pregnancy ${ }^{1,2}$, as well as by others ${ }^{3,4}$.

Alteration of methionine metabolism in humans due to folate or vitamin $B_{12}$ shortage may play a role in the aetiology of neural-tube defects, recurrent miscarriage, placental infarcts and placental abruption $^{2,5-9}$. The causes of these pregnancy complications might be found in the first gestational weeks. During the first trimester of pregnancy embryonic nutrition is provided by the transfer of nutrients from the

Correspondence: Dr R. P. M. Steegers-Theunissen, Department of Obstetrics and Gynaecology, University Hospital Nijmegen, PO Box 9101,6500 HB Nijmegen, The Netherlands. extra-embryonic coelomic and amniotic fluids to the embryo. Campbell et al. ${ }^{10}$ reported that the main route for maternal-fetal exchange of folate and methylcobalamin in early human pregnancy may be via the extra-embryonic coelomic cavity. Knowledge about the composition of the extra-embryonic coelomic and amniotic fluids and the transport mechanisms of methionine and homocysteine during that period of pregnancy is lacking.

Therefore the aim of the present study was to investigate the importance of tissue specific methionine metabolism during normal early human pregnancy by measuring the levels of methionine and total homocysteine in samples of maternal serum, extra-embryonic coelomic fluid and amniotic fluid at 8 to 12 weeks of gestation.

\section{METHODS}

Twenty-three pregnant women were studied after informed consent was obtained. The experimental protocol was approved by the Ethics Committee of St 


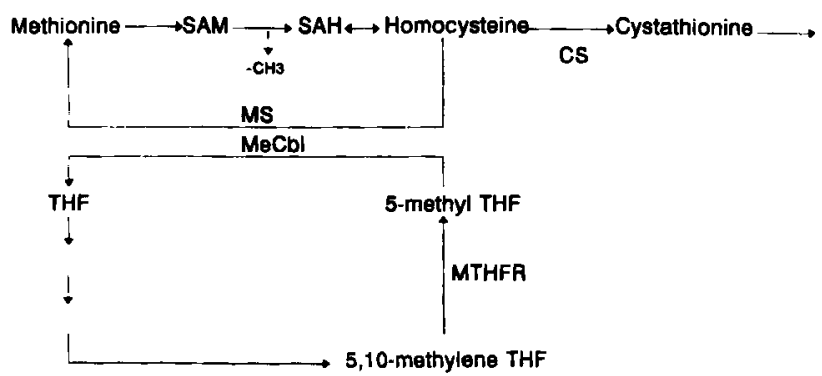

Fig. 1. A simplification of the folate and cobalamin dependent methionine metabolism in humans. $\mathrm{CS}=$ cystathionine synthase; $\mathrm{MeCbL}=$ methylcobalamin; $\mathrm{MS}=$ methionine synthase; MTHFR = methylene THF reductase; $\mathrm{SAH}=\mathrm{S}$-adenosylhomocysteine; $\mathrm{SAM}=$ S-adenosylmethionine; THF = tetrahydrofolate.

Bartholomew's Hospital, London. All women were undergoing a therapeutic termination of pregnancy for psycho-social reasons at a gestational age of 8 to 12 weeks. The duration of pregnancy was established by menstrual history and ultrasound measurement of crown-rump length.

Transvaginal ultrasound was performed using a $5 \mathrm{MHz}$ curvilinear vaginal probe (Aloka SSD 620, Aloka Co Ltd, Tokyo, Japan). In each case ultrasonography confirmed a singleton pregnancy with normal development and normal fetal heart activity. The procedure for transvaginal ultrasound, coelocentesis and amniocentesis has been described in detail ${ }^{11}$. Matched samples of amniotic and coelomic fluid were collected in every case.

Maternal blood was collected into glass tubes before induction of anaesthesia; within $30 \mathrm{~min}$, the samples were centrifuged for $10 \mathrm{~min}$ at $3000 \mathrm{~g}$ and the serum aspirated into dry plastic tubes. The fluid or serum was stored at $-20^{\circ} \mathrm{C}$ until assayed for total homocysteine (free plus protein-bound) and methionine. Total homocysteine concentrations were measured by automated high performance liquid chromatography and fluorometric detection in $70 \mu \mathrm{mol} / \mathrm{L}$ of the homogenate ${ }^{12}$. The total homocysteine determination by this method is based on complete reduction of all homocysteine disulfide bonds in plasma by sodiumborohydride and dithioerythritol. After derivatisation by monobromobimane the resulting homocysteine-monobromobimane complex was separated from interfering substances by reverse phase chromatography. The detection limit was $35 \mathrm{pmol}$ and the intra-assay coefficient of variation was $2 \cdot 1 \%$ and the inter-assay coefficient of variation was $5 \cdot 2 \%$. Methionine concentrations were determined according to a modified accelerated procedure on an automatic Biotronik LC 6001 (Biotronik, Frankfurt, Germany) ${ }^{13}$. The homogenates $(50 \mu \mathrm{L})$ were deproteinised by adding an equal
Table 1. Methionine levels in 23 matched samples of extra-embryonic coelomic fluid, amniotic fluid, and maternal serum at 8 to 12 weeks of gestation. Values are given as median (range).

\begin{tabular}{lcc}
\hline & Methionine $(\mu \mathrm{mol} / \mathrm{L})$ & Homocysteine $(\mu \mathrm{mol} / \mathrm{L})$ \\
\hline Serum & $11(2-20)$ & $8 \cdot 7(6-13 \cdot 1)$ \\
Coelomic fluid & $46(32-63)$ & $2 \cdot 5(0 \cdot 8-4 \cdot 2)$ \\
Amniotic fluid & $26(18-40)$ & $1 \cdot 0(0 \cdot 5-1 \cdot 7)$ \\
\hline
\end{tabular}

volume of ice-cold sulphosalicyclic acid $(25 \% \mathrm{w} / \mathrm{v})$ and were placed on ice. After $10 \mathrm{~min}$ the samples were centrifuged for $10 \mathrm{~min}$ at $3500 \mathrm{~g}$. The supernatant was filtrated through a $0.45 \mu \mathrm{m}$ filter. A $140 \mu \mathrm{L}$ sample was injected on a column. After post-column derivatisation with $\mathrm{O}$-phtaldialdehyde, the eluent was monitored fluorometrically. The detection limit was 15 pmol. Both intra- and inter-assay coefficients of variation were $<5 \%$.

Results have been expressed as median (range). Comparisons between gestational age, blood and fluid concentrations of methionine and homocysteine were performed by the Wilcoxon matched-pairs signedrank test. Correlations were evaluated by determination of the Spearman coefficient of correlation and careful interpretation of the scatter diagrams. $P$-values $<0.05$ were considered statistically significant.

\section{RESULTS}

The concentrations of methionine and total homocysteine in maternal venous blood, extra-embryonic coelomic fluid and amniotic fluid from 23 pregnancies at 8 to 12 weeks of gestation are shown in Table 1 .

Methionine levels were higher and homocysteine values were lower in extra-embryonic coelomic fluid and amniotic fluid when compared with maternal serum levels $(P<0.01)$. The highest methionine levels were found in extra-embryonic coelomic fluid and the lowest total homocysteine levels were found in amniotic fluid.

There were no relations between gestational age and methionine and total homocysteine concentrations in the various compartments (Fig. 2). All Spearman correlation coefficients were $r \leq 0.35$ and $P>0 \cdot 10$. Positive correlations could be established between methionine in extra-embryonic coelomic fluid and amniotic fluid (Spearman's coefficient of correlation $r=0.65, P<0.01$ ), total homocysteine in serum and extra-embryonic coelomic fluid $(r=0.56$, $P<0.01$ ), and between methionine in serum and total homocysteine in extraembryonic coelomic fluid $(r=0.43, P<0.05)$ (Fig. 3). 

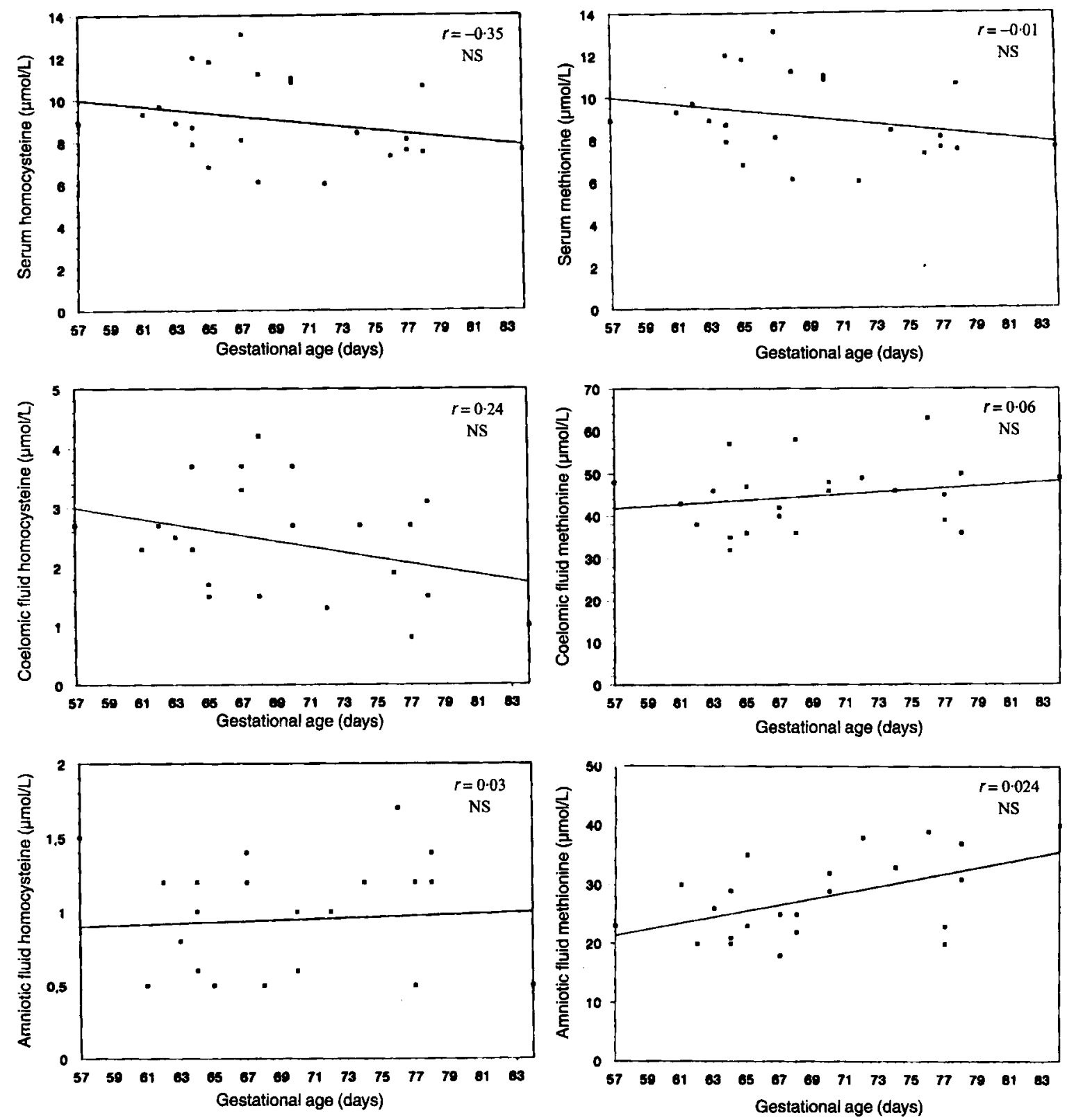

Fig. 2. The correlation coefficients between methionine and total homocysteine in the various compartments and gestational age. NS = not significant.

\section{DISCUSSION}

This is the first report on concentrations of methionine and total homocysteine in human extra-embryonic coelomic and amniotic fluids at 8 and 12 weeks of gestation. The comparatively high levels of methionine in both embryonic fluids may suggest that this nutrient is important during early human pregnancy.

Methionine is essential for cell proliferation and DNA and tRNA methylation. It is converted to Sadenosylmethionine and, after decarboxylation, this methyldonor is the source of the 3-carbon-moeities of the polyamines spermidine and spermine. In addition,
S-adenosylmethionine is involved in the methylation of DNA. In the rat a shortage of methionine and Sadenosylmethionine in embryo cultures can lead to disturbed morphogenesis, especially the development of neural tube defects ${ }^{14-17}$. The concomitant low total homocysteine concentrations in both fluids together with the high methionine concentrations suggests that the remethylation pathway is likely to be important as well in the extra-embryonic tissues during this early stage of development. Because folate and vitamin $B_{12}$ are essential in the remethylation of homocysteine into methionine, this hypothesis is supported by the results of the study of Campbell et al. ${ }^{10}$ showing high 

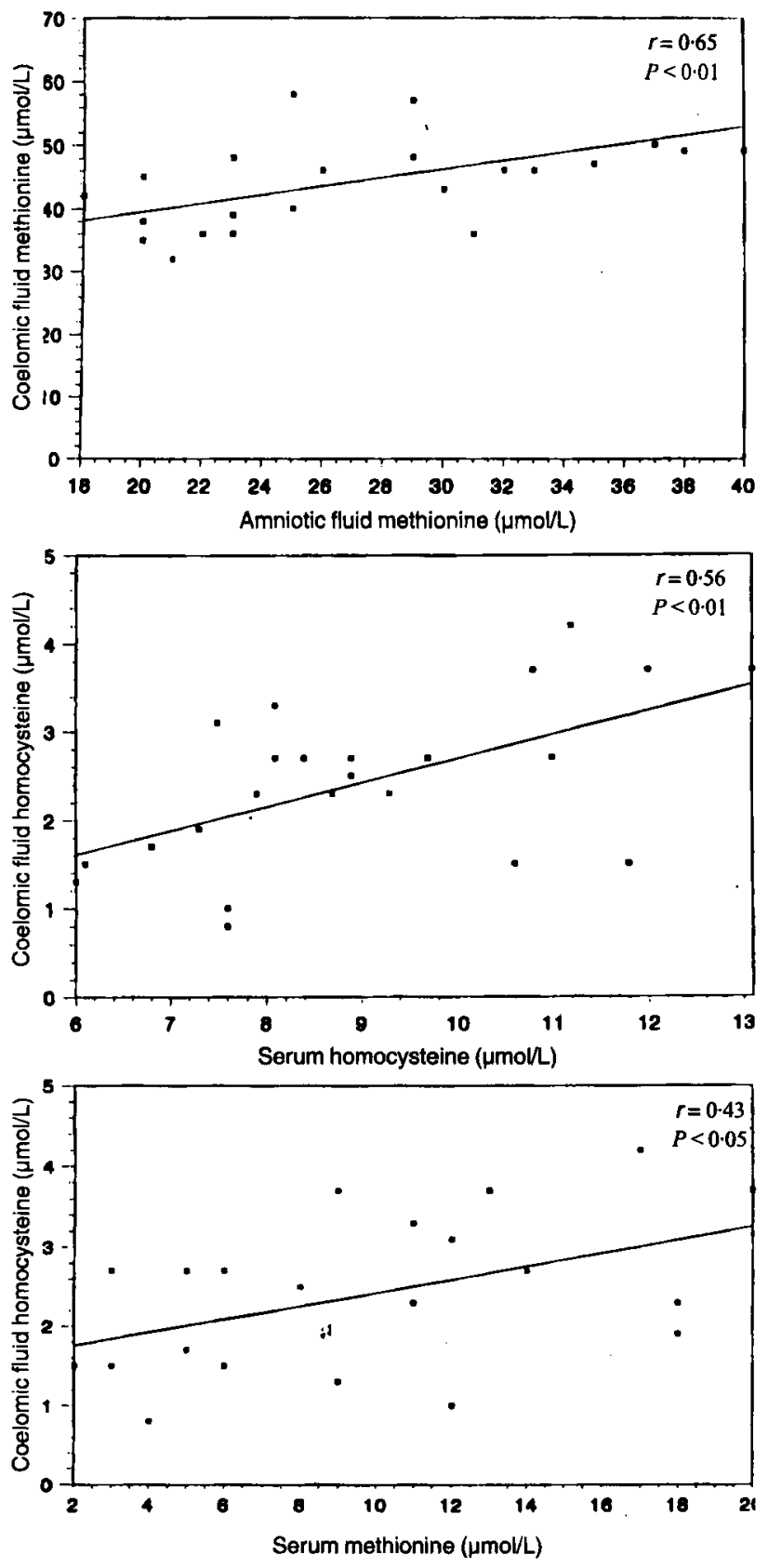

Fig. 3. The correlation coefficients between methionine and total homocysteine in various compartments.

levels of folate and vitamin $B_{12}$ in the extra-embryonic and amniotic cavity. High methionine may suggest that the coelomic cavity may act as a store of concentrated methionine prior to utilisation by the yolk sac and rapidly growing fetus and trophoblast. The gradient of methionine levels suggests active transport of methionine from maternal serum to the coelomic cavity followed by active transport or diffusion into the amniotic cavity. This is supported by the finding of a positive correlation between the methionine levels in the coelomic and amniotic fluids. The positive correlation between total homocysteine in serum and coelomic fluid might also be explained by a passive diffusion process.

The positive correlation between the methionine concentration in serum and the total homocysteine concentration in the coelomic cavity is more difficult to explain. It is possible that if large amounts of methionine are actively transported from maternal blood to coelomic fluid, then the remethylation of homocysteine to methionine by extra-embryonic structures would be decreased leading to relatively high total homocysteine concentrations in the coelomic fluid.

The methionine levels in the serum of pregnant women in the present study were slightly lower than those determined in nonpregnant women ${ }^{18}$, and the serum total homocysteine concentrations were slightly higher than those published previously by Andersson et al. ${ }^{19}$ in the first trimester of pregnancy.

The derivative homocysteine of methionine is normally present in blood in low concentrations. Elevated intracellular and extracellular homocysteine levels may be cytotoxic, though whether elevated circulating levels of homocysteine are embryotoxic is unknown $^{20}$. In vitro studies in the rat suggest that the embryotoxic effect of L-homocysteine is due to inhibition of methyl donation by S-adenosylmethionine ${ }^{21}$. Also, toxicity of homocysteine for vascular endothelium interfering with spiral or yolk sac arteries cannot be excluded. The development of neural tube defects might be partially explained by a decreased availability of methionine, folate and cobalamin, and subsequent derangement of methionine metabolism during early human pregnancy resulting in decreased synthesis of DNA and thus disordered cell proliferation. The prevention of neural tube defects by periconceptional folate supplementation might partly be explained by the correction of disturbed methionine metabolism ${ }^{6,8,22}$. This concept is supported by the results of the present study.

Although the supply, metabolism and synthesis of nutrients during early human pregnancy is poorly understood, the results of the present study suggest that folate and vitamin $\mathrm{B}_{12}$ dependent methionine metabolism may be important for growth and development of the human embryo.

\section{Acknowledgements}

The authors acknowledge the laboratory supervision of Dr H. Blom and the expert technical assistance of Mrs A. De Graaf-Hess of the laboratory of neurology and paediatrics, University Hospital Nijmegen, The Netherlands. 


\section{References}

1 Steegers-Theunissen RPM, Steegers EAP, Boer de R, Thomas CMG, Kloosterman MD, Eskes TKAB. Elevated folate levels in amniotic fluid after oral supplementation. Eur J Obstet Gynaecol 1990; 36: 283-298.

2 Steegers-Theunissen RPM, Boers GHJ, Blom HJ et al. Neural tube defects and elevated homocysteine levels in amniotic fluid. $\mathrm{Am} \mathrm{J}$ Obstet Gynecol 1995; 172: 1436-1441.

3 Berglund L, Halldin C, Lilja A et al. ${ }^{11} \mathrm{C}$-Methionine kinetics in pregnant rhesus monkeys studied by positron emission tomography: a new approach to feto-maternal metabolism. Acta Obstet Gynecol Scand 1984; 63: 641-645.

4 Kang SS, Wong PWK, Zhou J, Cook Y. Total homocyste(i)ne in plasma and amniotic fluid of pregnant women. Metabolism 1986; 35: 889-891.

5 Steegers-Theunissen RPM, Boers GHJ, Trijbels JMF, Eskes TKAB. Hyperhomocysteinaemia and recurrent spontaneous abortion or abruptio placentae. Lancet 1992; 1: 1122-1123.

6 Steegers-Theunissen RPM, Boers GHJ, Trijbels JMF et al. Maternal hyperhomocysteinaemia: a risk factor for neural tube defects? Metabolism 1994; 43: 1475-1480.

7 Wouters MGAJ, Boers GHJ, Blom HJ et al. Hyperhomocysteinaemia: a possible risk factor in women with recurrent early pregnancy loss? Fertil Steril 1993; 60: 820-825.

8 Mills JL, McPartlin JM, Kirke PN et al. Homocysteine metabolism in pregnancies complicated by neural-tube defects. Lancet 1995; 1: 149-151.

9 Kirke PN, Molloy AM, Daly LE, Burke H, Weir DG, Scott JM. Maternal plasma folate and vitamin B12 are independent risk factors for neural-tube defects. $Q J$ Med $1983 ;$ 86: 703-708.

10 Campbell J, Wathen N, Perry G, Soneji S, Sourial N, Chard T. The coelomic cavity: an important site of matemo-fetal nutrient exchange in the first trimester of pregnancy. Br J Obstet Gynaecol 1993; 100: 765-767.

11 Wathen NC, Cass PL, Kitau MJ, Chard T. Human chorionic gonadotrophin and alpha-fetoprotein levels in matched samples of amniotic fluid: extra-embryonic coelomic fluid and maternal serum in the first trimester of pregnancy. Prenat Diagn 1991; 11: 145-151.

12 Te Poele-Pothoff MT, Heijer den M, Franken DG et al. Three different methods for the determination of total homocysteine in plasma. Ann Clin Biochem 1995; 32: 218-220.
13 Gerrits GPJM, Trijbels JMF, Monnens LAH et al. Reference values for amino acids in cerebrospinal fluid of children determined using ion-exchange chromatography with fluorimetrie detection. Clin Chim Acta 1989; 182: 271-280.

14 Coelho CND, Weber JA, Klein NW, Daniels WG, Hoagland TA Whole rat embryos require methionine for neural tube closure when cultured on cow serum. $J$ Nutr $1987 ; 191$ : 1716-1725.

15 Coelho CND, Klein NW. Methionine and neural tube closure in cultured rat embryos: morphological and biochemical analyses. Teratology 1990; 42: 437-451.

16 Fujinaga M, Baden JM. Methionine prevents nitrous oxide-induced teratogenicity in rat embryos grown in culture. Anesthesiology 1994; 81: 184-189.

17 Seyoum G, Persaud TV. In vitro effect of S-adenosyl methionine on ethanol embryopathy in the rat. Exp Toxicol Pathol 1994; 46: 177-181.

18 Steegers-Theunissen RPM, Steegers EAP, Thomas CMG et al. Study on the presence of homocysteine in ovarian follicular fluid. Fertil Steril 1993; 60: 1006-1010.

19 Andersson A, Hultberg B, Brattström L, Isaksson A. Decreased serum homocysteine in pregnancy. Eur J Clin Chem Clin Biochem 1992; 30: 377-379.

20 Starkebaum G, Harlan JM. Endothelial cell injury due to copper-catalyzed hydrogen peroxide generation from homocysteine. $J$ Clin Invest 1986; 77: 1370-1376.

21 Aerts van LAGJM, Blom HJ, Abreu de RA et al. Prevention of neural tube defects by and toxicity of L-homocysteine in cultured postimplantation rat embryos. Teratology 1994; 50: 348-360.

22 Put van der NMJ, Steegers-Theunissen RPM, Frosst $P$ et al. Mutated methylenetetrahydrofolate reductase as a risk factor for spina bifida Lancet 1995; 2: 1070-1072.

Received 2 November 1995

Returned to Authors I February 1996

Resubmitted I April 1996

Accepted 9 July 1996 